\title{
Using A Digital Planetarium For Teaching Seasons To Undergraduates
}

Ka Chun Yu, Denver Museum of Nature \& Science, USA

Kamran Sahami, Metropolitan State University of Denver, USA

Victoria Sahami, Metropolitan State University of Denver, USA

Larry C. Sessions, Metropolitan State University of Denver, USA

\begin{abstract}
Computer-generated simulations and visualizations in digital planetariums have the potential to bridge the comprehension gap in astronomy education. Concepts involving three-dimensional spatial relationships can be difficult for the layperson to understand, since much of the traditional teaching materials used in astronomy education remain two-dimensional in nature. We study the student performance after viewing visualizations in an immersive theater and in non-immersive classrooms for the topic of seasons in an introductory undergraduate astronomy course. Using weekly multiple-choice quizzes to gauge student learning, comparison of curriculum tests taken immediately after instruction and pre-instruction quizzes show a significant difference in the results of students who viewed visualizations in the planetarium versus their counterparts who viewed non-immersive content in their classrooms, and those in the control group that saw no visualizations whatsoever. These results suggest that the immersive visuals help by freeing up cognitive resources that can be devoted to learning, while visualizations shown in the classroom may be an intrinsically inferior experience for students.
\end{abstract}

Keywords: Astronomy; Computer Simulation; Planetarium; Undergraduate Education

\section{INTRODUCTION}

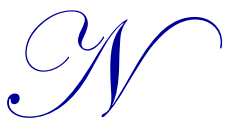

early 1300 digital "fulldome" video planetariums are now in operation worldwide (Lochness Productions, 2015). These venues project imagery onto the interior of a hemispherical dome, giving audiences an immersive visual experience (Lantz, 2011). Many of these digital theaters have interactive real-time software that recreate the universe as a simulated virtual environment (Emmart, 2005; Wyatt, 2005). These planetarium virtual environments allow audiences to gain direct experience about a place or phenomenon that would otherwise be difficult or impossible to observe in real life, much like their virtual reality counterparts (Dede 2000). Potential educational benefit from this technology comes from the ability to show threedimensional relationships, and accurate motions and lighting of astronomical bodies, from multiple frames of reference and at multiple scales (Yu, 2005).

Two aspects of the digital planetarium audience experience have been studied in other forms of media: exploring virtual models by using multiple perspectives, and the impact from physically immersive displays. For the first, the audience is placed at different positions inside the virtual model, which translate to different ways of navigation and exploration (Ware \& Osborne, 1990). An "egocentric" viewpoint allows the viewer to move through the model and view local detail from within, while an "exocentric" viewpoint gives global views from outside the model. Computer-generated visualizations that can show multiple frames of reference, including both egocentric and exocentric viewpoints instead of just a single perspective, have been shown to be important for learning in a variety of non-astronomy science topics (Salzman, Dede, \& Loftin 1999; Winn, Windschitl, Fruland, \& Lee, 2002; Jacobson 2011). Multiple frames of reference is also of benefit in astronomy education (Sadler, 1998). In studying daily celestial motions, elementary school students who receive instruction that is only Earth-based or only spacebased did not achieve the same level of understanding as students exposed to explanations that bridge the two frames (Plummer, Kocareli, \& Slagle, 2013). Cognitive research has shown that visually immersive egocentric 
exploration has distinctly different impacts when compared with a non-immersive exocentric experience (Kozhevnikov \& Garcia, 2011). In the egocentric case, virtual object locations and orientations are processed by the user to be relative to a body-centered reference frame. In comparison, the exocentric or "allocentric" viewpoint is centered on a stationary environmental frame of reference, and defined relative to another object, such as a computer screen or room. These two viewpoints require different cognitive encoding and spatial transformation processing by a viewer (Kozhevnikov \& Dhond, 2012). Training that requires egocentric navigation and spatial transformation strategies are done most effectively with immersive virtual environments (Kozhevnikov \& Garcia, 2011).

Non-immersive astronomy virtual environments created for desktop computers have been shown to be effective in increasing student understanding of motions of the Sun and Earth (Bakas \& Mikropoulos, 2003; Chen et al., 2007), and phases of the Moon (Trundle \& Bell, 2010; Hobson, Trundle, \& Sackes, 2009). Simulations that allow users to interact and model their virtual environments can help increase student understanding of abstract spatial concepts in astronomy (Keating et al., 2002; Hansen et al., 2004). When high school students were given the controls to one virtual solar system simulator for guided self-exploration, they were able to transfer their understanding of the concept of day-night from the Earth to the Moon (Gazit, Yair, \& Chen, 2005).

A number of immersive virtual environments have been tested for astronomy education with generally positive results. One showed limited success in teaching the shape of the Earth (Johnson, Moher, Ohlsson, \& Gillingham, 1999). In another study, elementary students showed similar improvements when taught in an immersive planetarium dome, versus commercial planetarium software on a desktop computer (Baxter \& Preece, 2000). A combination of planetarium visuals with kinesthetic activities resulted in increased understanding of celestial motions by elementary school children (Plummer, 2009; Plummer \& Krajcik, 2010; Plummer et al., 2013). A portable digital planetarium by itself was effective for increasing understanding of lunar phases by middle schoolchildren (Chastenay, 2015). Many digital planetarium programs in museums primarily consist of playback of pre-rendered films, instead of a live presentation by a speaker. Focusing on this paradigm, Sumners, Reiff, \& Weber (2008) and Zimmerman, Spillane, Reiff, \& Sumners (2014) showed that short ( 25 minutes long) movies were effective at increasing student knowledge about Earth science and human spaceflight, while Heimlich et al. (2010) showed that a 35 minute fulldome movie about Maya astronomy was effective for increasing a host of learning, perceptual, and engagement factors in museum visitors. Although these studies used immersive visualizations, only Heimlich et al. investigated the roles that immersion and non-immersion played in audience gains. The contribution from the other aspects of the learning experience (those unrelated to immersion) in the other studies is unclear.

Our current work expands on these efforts by comparing how an astronomy virtual environment in an immersive planetarium can assist college undergraduate students in learning about Earth's seasons. Our approach is informed by Fraser et al.'s (2012) hypothesis that visual immersion from a giant screen cinema or fulldome planetarium results in the viewer becoming absorbed by the visuals and story content, which heightens the learning experience. For our curriculum design, we adopt a constructivist framework, whereby a learner holds a privately held mental model, informed by prior information and experience, and which may contain alternative conceptions, ideas that are at odds with accepted scientific thinking (Brewer, 2008). Newly acquired scientific information can be combined with existing alternative conceptions to create synthetic models (Vosniadou \& Brewer, 1994). As the student continues to learn, the model can evolve to be more scientifically correct. An alternative conception that has taken hold in the mind of a learner can be difficult to dislodge (Sadler, 1998), meaning the transformation of mental models to scientific form can be very gradual. Introducing conflicting evidence can help promote conceptual change (Scott, Asoko, \& Driver, 1992; Bakas \& Mikropoulos, 2003).

\section{Astronomical Instruction on Seasons}

Alternative conceptions on the cause of seasons on Earth have been studied mainly in grade school students (e.g., Baxter, 1989; Kikas, 1998). University students show similar alternative conceptions - such as the seasons being the result of the Sun-Earth distance, or the Sun being directly overhead in the sky at noon at mid-latitudes (Bisard, Aron, Francek, \& Nelson, 1994; Zeilik, Schau, \& Mattern, 1999; Trumper 2000). Synthetic mental models for why it is colder in winter include: our side of Earth rotates away from the Sun; Earth is further in its orbit from the Sun; and the Earth "tilts" away during winter. Textbook diagrams may also be directly responsible for erroneous ideas (Ojala, 1992, 1997) such as: the equatorial regions on the Earth are significantly closer to the Sun than the 
polar regions; the Earth's inclination relative to the ecliptic causes each hemisphere to tilt substantially closer to or further from the Sun during summer or winter; the Earth's orbit is much more eccentric than it really is; and the ratio of the Earth's size to that of the Sun's is greatly exaggerated. Some of these nonscientific ideas are also found among adults including pre-service teachers, with the Earth-Sun distance the most pervasive alternative conception (Bisard et al., 1994; Atwood \& Atwood, 1996).

The ease by which alternative conceptions about the seasons take hold can be explained in part by the complexity of the scientific model: a large number of concepts need to be understood before a learner can have a complete conceptual understanding (Willard \& Roseman, 2007; Sneider, Bar, \& Kavanagh, 2011). Sub-concepts identified by Sneider et al. and Lucas \& Cohen (1999) include: Earth is spherical; Earth's axis is tilted with respect to its orbit, and its orientation remains constant; Earth's orbit is eccentric; Earth is nearer the Sun during the northern winter; Earth's motion and orientation translate to the changing appearance of the Sun over the course of a year in the sky for an Earth-bound observer (e.g., the Sun appears higher in the sky in the summer and lower in the winter, and the Sun rises and sets in different cardinal directions over the course of the year); seasons are more pronounced at higher latitude; the length of day and night varies by latitude and throughout the year; the amount of solar radiation on Earth's surface varies by latitude; the amount of light and heat from the Sun affects Earth's climate zones; and the onset and the intensity of seasonal effects can vary by location.
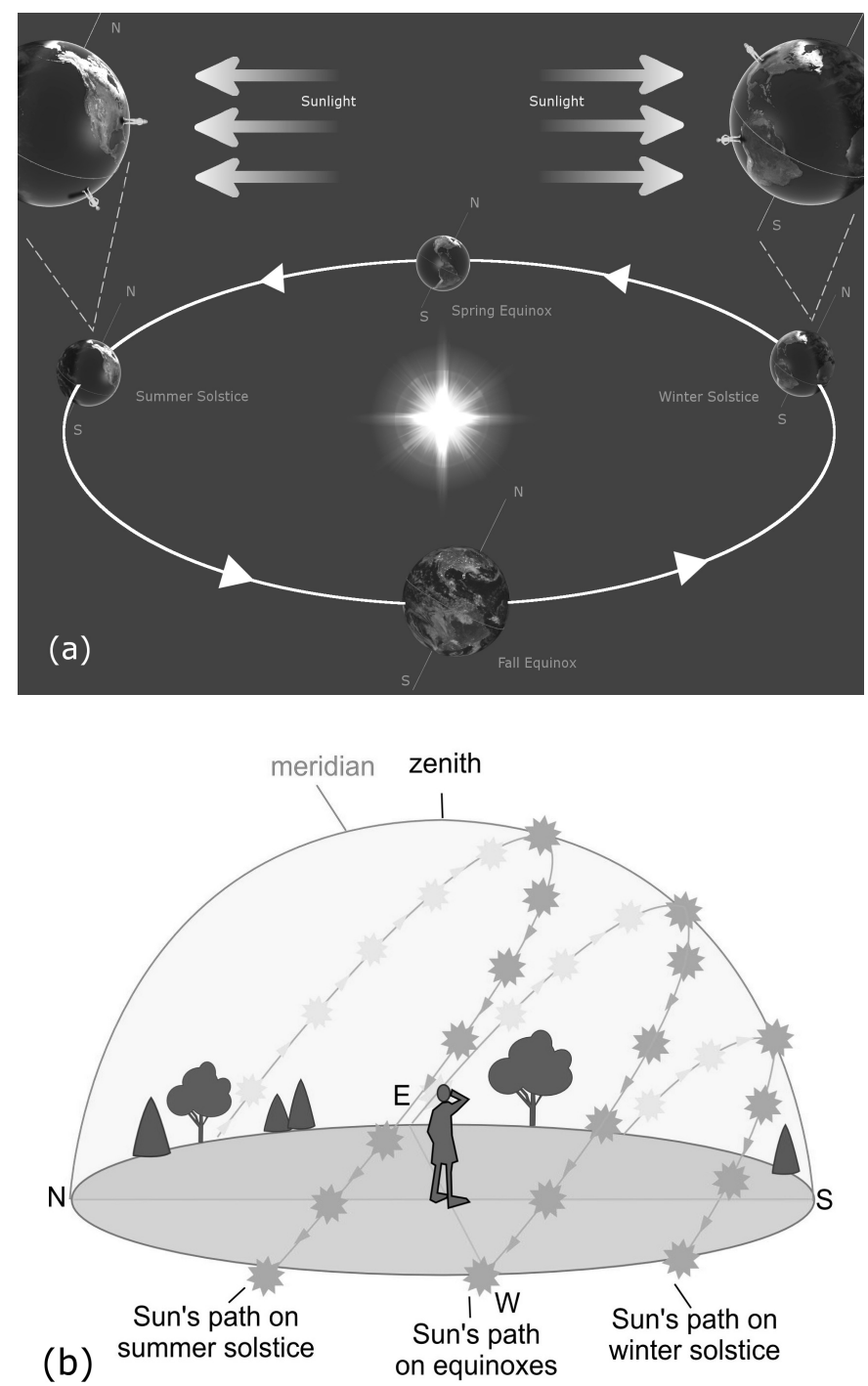

Figure 1. Diagrams for seasons instruction similar to those in Bennett et al. (2007). 
Simplifications in textbook diagrams can also lead to problems in understanding. In Figure 1a (based on the one in the textbook used by students in this study, Bennett, Donahue, Schneider, \& Voit, 2007), multiple instances of Earth are shown in orbit around the Sun, with expanded views of Earth at the top of the figure showing sunlight's effects on people at different latitudes and the shadows that result. The different paths of the Sun across the sky from a ground-view are shown in a second diagram (Figure 1b, again based on one from Bennett et al.). Such diagrams compress a dynamic three-dimensional (3D) system into a static two-dimensional (2D) view (Parker \& Heywood, 1998; Peña \& Quilez, 2001), and students are asked to conceptualize the 3D abstractions using the 2D descriptions (Subramaniam \& Padalkar, 2009). In Figure 1b, students have to conceptually link what they experience from an Earth-bound perspective with the space-based viewpoint of Figure 1a. Spatial visualization skills (including understanding $3 \mathrm{D}$ relationships from $2 \mathrm{D}$ representations, ability to imagine how an object appears from a different perspective, and the ability to mentally rotate objects; Barnea \& Dori, 1999) correlate with the amount of conceptual astronomy knowledge in students (Heyer, 2013), and may explain differences recorded in student understanding of astronomy (Plummer and Maynard, 2013; Plummer et al. 2013).

\section{METHODOLOGY}

Astronomy Learning in Immersive Virtual Environments (ALIVE) is a collaborative research project between the Denver Museum of Nature \& Science (DMNS) and the Metropolitan State University of Denver (MSUD), with the main goal of answering the question, is there a difference in student understanding of introductory astronomy when taught with immersive and non-immersive versions of the same visualizations? Used for test subjects were undergraduate students enrolled in MSUD's AST1040, which covered topics in Solar System, galactic, and extragalactic astronomy. The four-year study was broken up into developmental and experimental phases. The former included prior-to-instruction oral interviews with more than 120 students enrolled in the Fall 2005 and Spring 2006 AST1040 courses (Yu, Sahami, \& Denn, 2010). Analysis of these front-end evaluations informed the creation of new lecture outlines to directly address common student alternative conceptions in seven different astronomical content modules (lunar phases, eclipses, seasons, Kepler's laws and orbits, scale of the Solar System, outer moon systems, distances to stars and galaxies). A suite of interactive visualization modules was developed by the authors following these outlines using the virtual environment software, SCISS AB's Uniview ${ }^{\mathrm{TM}}$. The design of the seasons instructional module was developed independently, but matched the learning progression of Willard \& Roseman (2007) based on benchmarks identified by AAAS (2007), including switching between Earth and space-based reference frames. A summary of the concepts introduced in the lecture and the instructors' lecture outline can be found in Appendix A.

Seventeen AST1040 classes were divided into three groups: Group I saw no visualizations; Group II students were exposed to instruction using visualizations projected onto a flat screen in the classroom; Group III students experienced instruction using immersive versions of the same visualizations projected in DMNS' Gates Planetarium. Classes in all three groups used the same textbook, and were presented with the same lectures for a particular topic, following the same outline, and at the same pace. Additional instructional content for the seven topics being investigated in ALIVE was integrated into all classes. To avoid confounding the impact of the visualizations, no other animations or visual media about a topic investigated in the study were shown in any of the classes. Beyond the visualizations, any differences between the students' experience in the classes were the result of classroom participation only. Instead of visualizations from the Uniview software, Group I students were exposed to PowerPoint presentations showing the two diagrams on seasons from the Bennett et al. textbook shared by all three Groups (which Figures 1a and $1 \mathrm{~b}$ are facsimiles of ), as well as a demonstration from the instructor using a physical globe. Transit time from the MSUD campus to the Gates Planetarium was approximately 10 minutes, and students boarded busses 5 minutes before the start of the 75 minute class. The visualizations were the same for both Groups II and III, and took up approximately 60 minutes including discussions. Hence the time spent in transit did not impact the amount of instruction for students who traveled to the planetarium.

The schedule for which classes would fall under Groups I, II, and III was determined with the individual instructors in the months prior to the start of each semester. The only exceptions were the control Group I classes, which took place in the first year of the project to allow for additional time to finalize the development of the visualization modules. The students did not learn about the study, nor which experimental group their class was in, until the first day of classes. Those who chose to participate were informed of their rights, and filled out a consent 
form and a demographics survey. A total of 810 students gave their consent: $57 \%$ were male and $43 \%$ female, with the age breakdowns: $45 \%$ for $15-20$ year olds, $36 \%$ for $21-25$ year olds, $12 \%$ for $26-30$ year olds, $6 \%$ for $31-35$ year olds, and $3 \%$ for 36 years and older.

\section{Visualizations in the Planetarium and Classroom}

The visualization modules were presented over four different days during the semester, with two topics covered in each of the first three days, while the fourth day was devoted to instruction of only a single topic module. The seasons module was paired with instruction on orbits and Kepler's laws of planetary motion. The Uniview software was controlled live by a navigator for each visualization session, while the instructor lectured following the same lecture outline for each class. Software features allowed camera position and orientation, simulation time, and other presets to be saved, so that the visualization scenes designed for the learning objectives could be repeated for each class. The software also made it possible to diverge from the script, such as going through a scene faster or slower, or repeating a scene. However because of time constraints, the sequence of visuals was usually strictly followed for each of the topics.

The Gates Planetarium dome is $17 \mathrm{~m}$ in diameter and is tilted by $25^{\circ}$ (Figure 2). The seating matches the dome tilt, with each seat row on a stepped floor surface, allowing all of the audience to have an unobstructed view of the front of the dome. The unidirectional seating also means that all 120 audience members view and share in a very similar immersive visual experience.
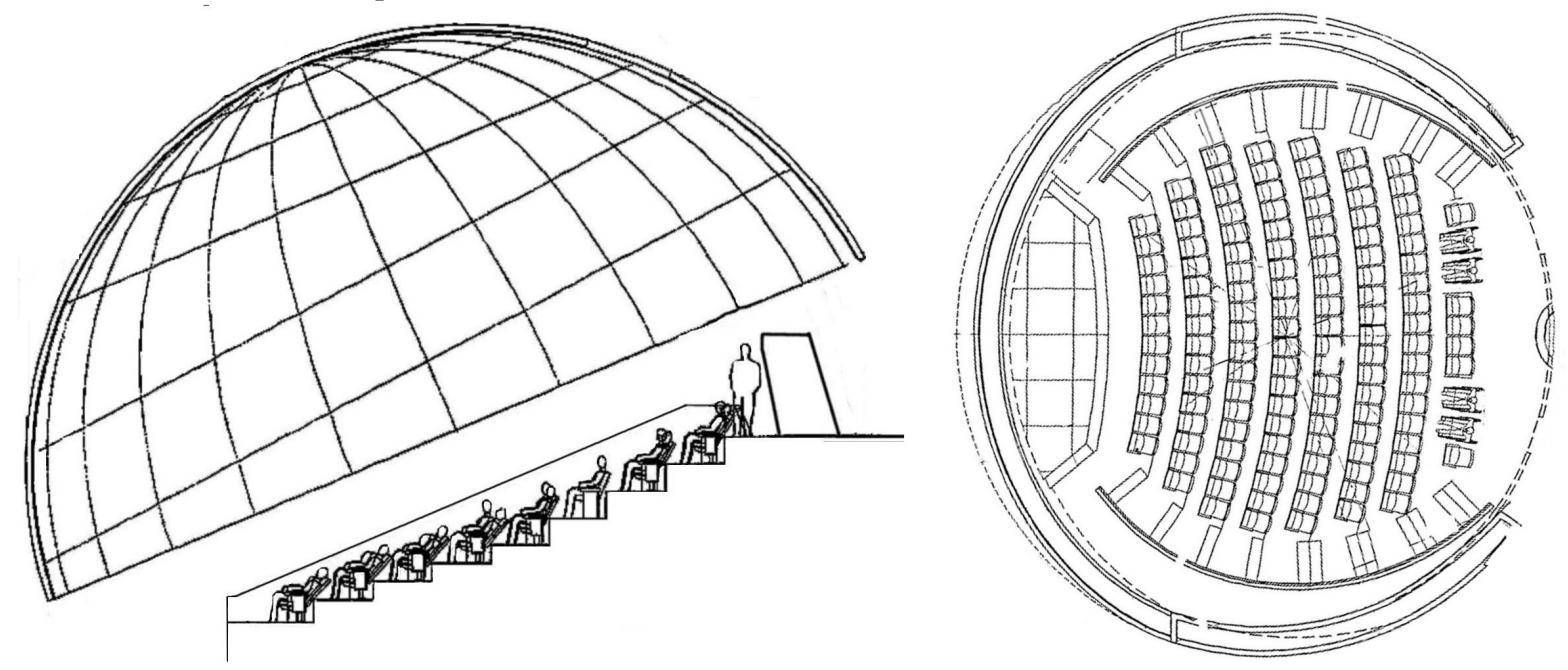

Figure 2. A profile (Left) and plan (Right) view of the Gates Planetarium. In both views, the front of the dome is to the left, and the back to the right.

Students in a Group II class saw the visualization modules projected at XGA $(1024 \times 768)$ resolution onto a flat display screen in one of two classrooms. The screens were $2 \mathrm{~m}$ and $2.3 \mathrm{~m}$ across, with the classroom seating organized such that students sat 3-12m away, with the display subtending an angle $11-29^{\circ}$. Like the planetarium, the classroom lighting was dimmed so that student attention could be focused on the projected visuals. Group I classes were in the same rooms, and had normal lighting that was not dimmed.

\section{Curriculum Tests}

The authors and two other professors at MSUD's Physics Department (all six of whom have extensive teaching and research experience in astronomy) developed a test bank of multiple choice questions for use in the weekly curriculum tests, after analyzing the pre-instruction student interviews, and reviewing existing literature (including those used to develop the Astronomy Diagnostic Test; Hufnagel, 2002). The quizzes contain questions that act as a pre-test for upcoming lectures, cover current instruction, and test retention of knowledge from earlier instruction. None of the questions were repeated between the three categories, meaning students could not have 
simply memorized the answers to an earlier test for a later quiz. Popular alternative conceptions were used as distractors, a technique used to identify such conceptions in the test-takers (Sadler, Sonnert, Coyle, Cook-Smith, \& Miller, 2013). A typical quiz contained questions for all topics covered in the class, in addition to those selected from the test-bank specifically for the ALIVE topic modules. The same five pre-instruction, nine contemporaneous, and five post-instruction questions covering seasons were used for every class in the experiment. Kuder-Richardson 20 (KR20) analyses show a reliability of $0.429,0.590$, and 0.429 for respectively, the pre-, contemporaneous, and post-instruction questions, which are good values for reliability for tests with such few questions. (These questions can be found in the Appendix B.)

A total of twelve curriculum quizzes were given during the 15 week course, which were cumulatively worth $40 \%$ or $45 \%$ (depending on the instructor) of the final class grade. The seasons pre-instruction questions were given at the end of Week 1 . The contemporaneous questions were given typically at the end of Week 2, although in three of the classes, they were in Week 3, and in one class, in Week 4. The post-instruction questions were given in either Weeks 7, 8, or 9, with 6 weeks being the median separation time between the contemporaneous and postinstruction questions.

Table 1 lists the number of MSUD classes and the number of students contributing data to the preinstruction, contemporaneous, and post-instruction quiz results. The three Group I classes took place in the first year of the experiment in Fall 2006. Group II and III classes were distributed from the Spring 2007 through Spring 2010 semesters. The course load was divided evenly between the two instructors (authors L. Sessions and V. Sahami), with both instructors covering classes in all three experimental groups, and results from both instructors aggregated together for analysis.

Table 1. The number of AST1040 classes and students participating in the experiment for Groups I, II, and III.

\begin{tabular}{lcccc}
\hline & Group I & Group II & Group III & Total \\
\hline Pre $\mathrm{n}$ & 52 & 160 & 336 & 548 \\
Cont $\mathrm{n}$ & 126 & 195 & 322 & 643 \\
Post $\mathrm{n}$ & 103 & 177 & 275 & 555 \\
No. of Classes & 3 & 5 & 9 & 17 \\
\hline
\end{tabular}

\section{RESULTS}

Table 2 lists the means $(M)$ for seasons-related questions on the curriculum quizzes for the classes in the three experimental groups. We tabulate the percentage test score gains between the contemporaneous and preinstruction quizzes (Cont-Pre), the post- and pre-instructional quizzes (Post-Pre), gains measured in effect sizes (ES; Cohen, 1977), and the $95 \%$ confidence interval (CI). We choose to display our results in terms of effect sizes and CIs instead of citing $p$ values from null hypothesis significance testing (NHST) because of the ease of misinterpretation of the latter method (Cohen, 1994; Kline, 2004). Among other benefits, the use of CIs gives point and interval estimates that are easily understandable within a research context, can be converted into $p$ values, and can be adopted for future meta-analysis studies (Cumming \& Finch, 2001). 
Table 2. The mean score (M), number of students (n), standard error (SE), and $95 \%$ confidence intervals (CI) in the preinstruction, contemporaneous, and post-instruction curriculum quizzes, along with relative gains between contemporaneous and pre-instruction scores, and post- and pre-instruction scores expressed in percentages and effect sizes (ES).

\begin{tabular}{lcccc|cc|cc}
\hline & \multicolumn{9}{c}{} & \multicolumn{2}{c}{ Gains } & \multicolumn{2}{c}{ ES } \\
\hline Group I & & Pre & Cont & Post & Cont-Pre & Post-Pre & Cont-Pre & Post-Pre \\
& $M$ & $51.5 \%$ & $64.0 \%$ & $66.0 \%$ & $4.4 \%$ & $16.7 \%$ & 0.174 & 0.556 \\
& $n$ & 52 & 126 & 103 & 44 & 39 & 44 & 39 \\
& $S E$ & $3.8 \%$ & $2.2 \%$ & $3.1 \%$ & $4.1 \%$ & $5.5 \%$ & 0.161 & 0.183 \\
& $95 \% \mathrm{CI}$ & $7.7 \%$ & $4.3 \%$ & $6.1 \%$ & $8.0 \%$ & $10.7 \%$ & 0.325 & 0.370 \\
\hline Group II & $M$ & $57.8 \%$ & $63.8 \%$ & $63.3 \%$ & $6.7 \%$ & $3.4 \%$ & 0.270 & 0.125 \\
& $n$ & 160 & 195 & 177 & 139 & 129 & 139 & 129 \\
& $S E$ & $2.0 \%$ & $1.7 \%$ & $2.1 \%$ & $2.6 \%$ & $3.0 \%$ & 0.103 & 0.112 \\
& $95 \% \mathrm{CI}$ & $4.0 \%$ & $3.4 \%$ & $4.1 \%$ & $5.0 \%$ & $6.0 \%$ & 0.204 & 0.222 \\
\hline Group III & $M$ & $47.5 \%$ & $64.8 \%$ & $68.4 \%$ & $17.4 \%$ & $20.9 \%$ & 0.696 & 0.741 \\
& $n$ & 336 & 322 & 275 & 294 & 260 & 294 & 260 \\
& $S E$ & $1.4 \%$ & $1.3 \%$ & $1.8 \%$ & $1.7 \%$ & $2.3 \%$ & 0.068 & 0.082 \\
& $95 \% \mathrm{CI}$ & $2.8 \%$ & $2.6 \%$ & $3.6 \%$ & $3.3 \%$ & $4.6 \%$ & 0.133 & 0.162 \\
\hline
\end{tabular}

The value of immersive visualizations is evident since the Group III gains ( $E S=0.696$ for Cont-Pre; $E S=0.741$ for Post-Pre gains) are larger than comparable gains in Groups I $(E S=0.174 ; E S=0.556)$ and II $(E S=0.270 ; E S=0.125)$. When comparing Cont-Pre questions, the Group III effect size is more than twice that of Group II ( $E S=0.696$ versus 0.270$)$. Group II students have greater learning gains than Group I classes, although the effect sizes for both groups are small $(E S=0.270$ versus 0.174$)$. However this advantage not only disappears but reverses in the Post-Pre results. Students in the control classes have learning gains with a modest $E S=0.556$, while the Group II students have dropped to a small $E S=0.125$. Group III students have increased their gains since the contemporaneous quizzes to $E S=0.741$.

In Figure 3, we plot the Cont-Pre gains for the three experimental groups. Following Cumming and Finch (2005), we plot CIs instead of $S E$ bars. The CI bars show the plausible range of values for the population mean for these measures. Due to sampling variability, only $83.4 \%$ of replications of the same experiment will result in a measured mean that falls within the CI interval (Cumming \& Maillardet, 2006). Within the context of NHST, if a null hypothesized value lies inside the $95 \% \mathrm{CI}$ bar, the null hypothesis cannot be rejected with a two-tailed test at the .05 level; if it lies outside the interval, it can be rejected at the .05 level (Cumming, 2007). From Figure 3, the Group III CI does not overlap with either the Group I or II CIs, meaning that $\mathrm{p}<0.01$ when considering the null hypothesis that either the Group I or II mean is outside the Group III CI (Cummings \& Finch, 2005). Although the Group II Cont-Pre gains was $6.7 \%$ versus $4.4 \%$ for Group I, the $95 \%$ CIs for the two groups overlap, meaning $\mathrm{p}>0.05$ when considering the null hypothesis that the Group II mean is within the Group I CI, and vice versa. 


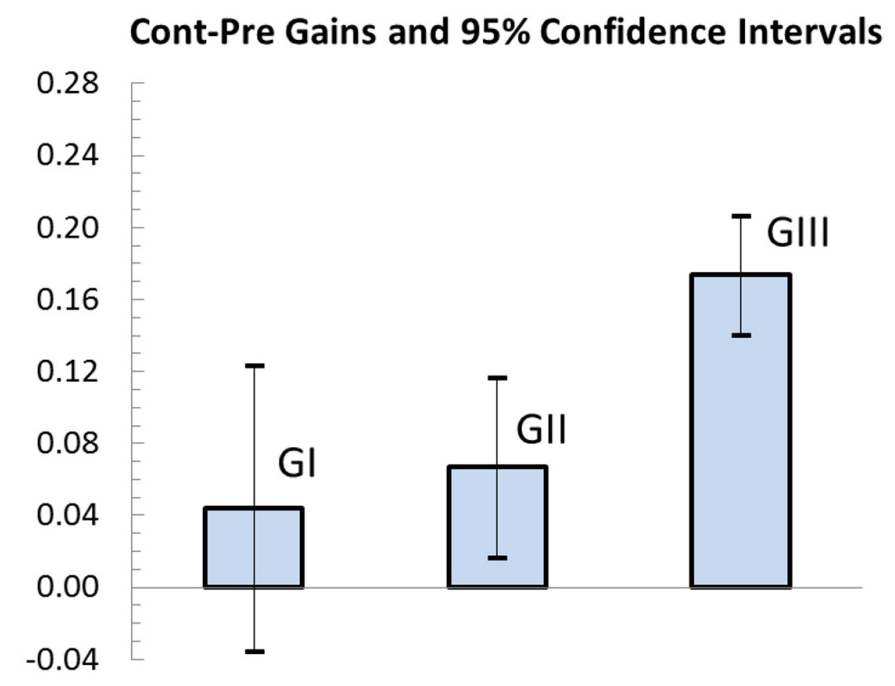

Figure 3. Gains between contemporaneous and pre-instruction quizzes, plotted with $95 \%$ confidence intervals.

Figure 4 shows the Post-Pre gains. The greater Group III gain is robust when compared with Group II since their 95\% CIs do not overlap. However the Group I CI is wide enough to encompass the Group III CI because the number of Group I students was lower. Although $n=52,126$, and 103 for the Pre-, Cont-, and Post- categories, respectively, the number of Group I students present for both the Post- and Pre- quizzes was only $n=39$, leading to a CI of $\pm 10.7 \%$. In comparison, there were more Group III students $(n=260)$ who were present for both the Post- and Pre- quizzes, resulting in a smaller CI of $\pm 4.6 \%$. This illustrates that the Group III gains are not only greater, but more reliable.

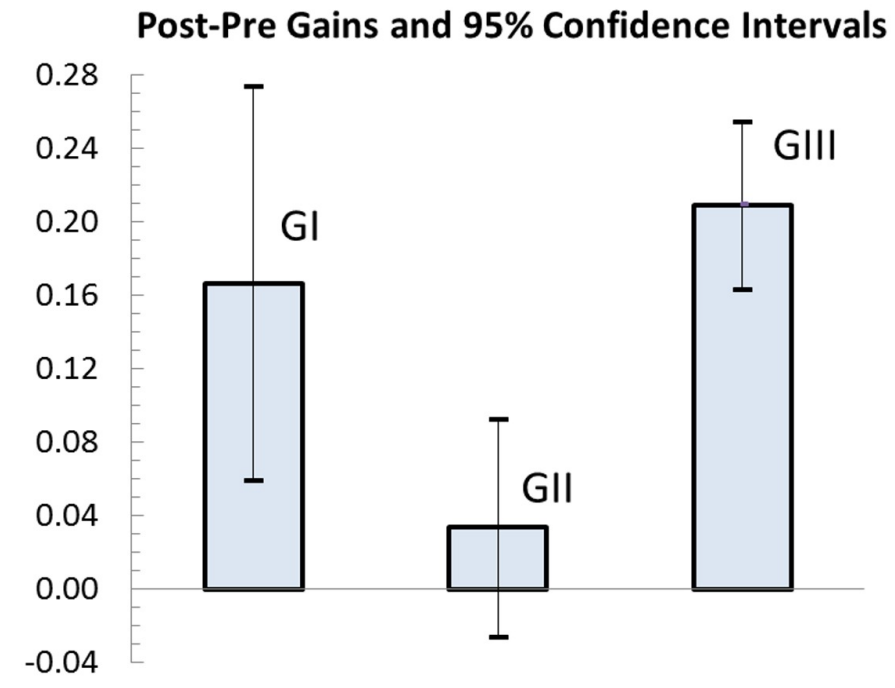

Figure 4. Gains between post-instruction and pre-instruction quizzes, plotted with $95 \%$ confidence intervals. 


\section{DISCUSSION}

Group III students who experienced visualizations in the immersive planetarium generally performed better than their counterparts in the other Groups. What in the student experience might account for these different learning gains suggested by the quiz results? We identify two aspects below.

\section{Benefits from the Virtual Environment}

The use of a virtual environment, common to both Groups II and III, has three main benefits. First it can accurately represent the true scale, orientation, and position of Solar System objects, meaning that anomalous information which contradicts commonly held beliefs can be presented. For the synthetic mental model that the seasons are caused by Earth's distance from the Sun, Earth's orbit is compared to a circle with a radius equal to the mean Earth-Sun distance. Even with its small eccentricity, Earth's orbit can be shown to deviate from a circle, so that even in the Group II classroom display, Earth appears further from the mean distance in July, and closer than the mean distance in January. Anomalous data by itself may not actually result in a rejection of a deeply held incorrect conception by the learner (Chinn \& Brewer, 1998). Yet the introduction of such discrepant data can help start the process of conceptual change (Limon \& Carretero, 1997; Mason, 2000).

Second, the virtual environment can show different frames of reference, a strategy prescribed in the seasons learning progression by Willard \& Roseman (2007). The lecture on seasons intersperses Earth-bound views of the sky from different latitudes with orbital views looking back at Earth, while the instructor connects the two different sets of perspectives in the oral presentation. These viewpoints bridge the familiar Earth-based experience of the seasons with the space-based depiction found in textbooks, and are consistent with Plummer et al.'s (2013) finding that showing multiple perspectives provide greater benefits for schoolchildren learning about daily celestial motions. In contrast, Group I students who only see 2D representations like those in Fig. 1 may not get the instructional support they need for comprehension if they have inadequate spatial visualization skills.

Finally the virtual environment shows time variable phenomena, such as how the altitude of the Sun and length of day change throughout the year. The daily motion and changes in apparent solar trajectory over the course of a year are readily discernible in the virtual environment visualizations. Group I students however are again relying on only static diagrams and verbal descriptions to model these motions.

\section{Limitations of Non-Immersive Visualizations}

In Figures 3 and 4, students viewing the astronomy simulation in the immersive digital dome had consistently larger gains than those who viewed the non-immersive version in their classroom. Why would the same virtual environment shown following the same lecture outline by the same instructors lead to such disparate results?

Part of the explanation may be related to the immersive medium itself. Although both Groups II and III get an exocentric view of Earth from space, only the Group III students in the planetarium have an egocentric experience surrounded by the visuals. These immersive visualizations act as a memory aid of where the Sun is, how high it has risen, and its sunrise and sunset directions. The students' cognitive resources can be used to organize the verbal and visual information to construct knowledge about the seasons. Group II students must use the visuals in the non-immersive classroom display and the instructor's lectures to reconstruct the location of the Sun. If they use up the available short-term memory resources in doing so, then learning about the cause of the seasons will not occur (Sweller, Van Merrienboer, \& Paas, 1998). In Hobson et al.'s study (2010), much younger (7-9 years old) learners benefited from planetarium visualization software, with the authors also arguing that computer simulations freed up cognitive capacity allowing the students to construct the correct scientific model for lunar phases.

The classroom display is also visually inferior to the experience in the immersive digital planetarium. As noted earlier, the virtual environment display in the classroom subtended $11-29^{\circ}$, depending on where a student was seated. In addition to the small apparent size of the screen, the imagery projected showed only a fraction of the hemispherical imagery visible in the planetarium. There is a trade-off between the field-of-view (FOV) of the rendered video and the detail that can be discerned from the projected imagery, given the fixed resolution of the 
classroom projector. Thus if the virtual scene is rendered out to show a larger FOV, the video output is still being projected from a XGA display to the same subtended angle as seen by a student. In order for a XGA projection to show the Sun at its highest point in the sky from Denver at the June solstice and keep the horizon in frame, the rendered FOV has to be $98^{\circ}$ across, meaning a large FOV can result in visual details rendered too small to be easily seen and comprehended in a flat display, when compared with the same visuals seen in the planetarium (Figure 5a). Conversely the FOV can be smaller to match the resolution of the planetarium display, and to keep the details visible in the classroom (Figure 5b). But since less of the visual content is seen simultaneously, a viewer loses context of where the Sun is in the sky. If the virtual camera pans from east to west to follow the Sun's motion in the Group II classroom, the learner cannot easily tell how far the camera has moved or how high above the horizon the Sun travels.

The virtual camera in the dome, on the other hand, can remain static during the demonstration of solar motion. The hemispherical display, with a physical FOV of $180^{\circ}$, can show widely separated spatial phenomena with great detail, and requiring no (or minimal) virtual camera motions (Figure 5c). Learners can simply move their heads to follow the virtual scene unfold around them. Thus keeping track of the relative position of the Sun in the sky is trivial in the immersive presentation, but more difficult in the flat screen version with a limited FOV and moving virtual camera.
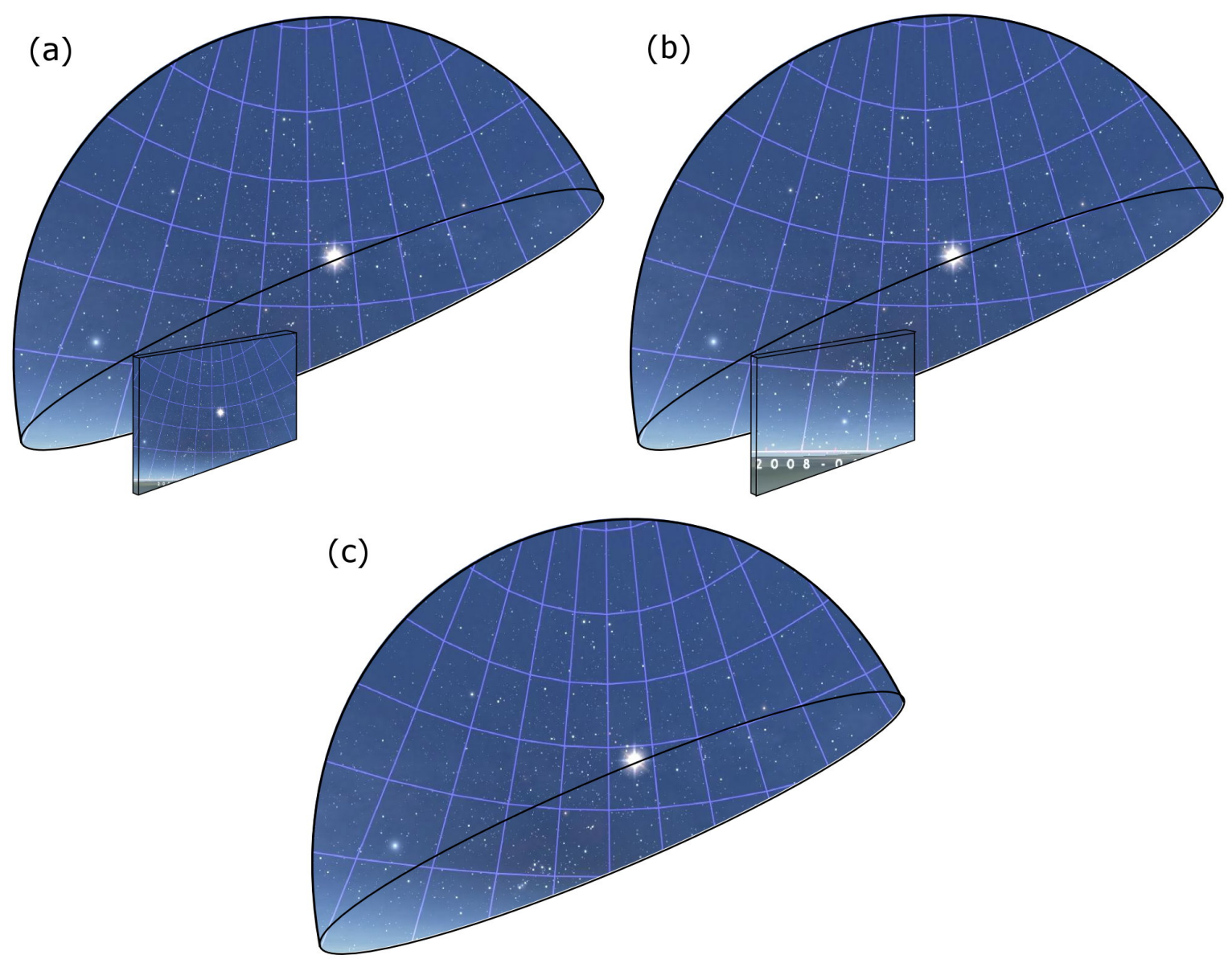

Figure 5. Comparing the experiences of students in Groups II and III. (a) A flat presentation in the classroom may show a large field-of-view (FOV), which results in a loss of detail compared to the dome experience. (b) Alternatively the FOV can be made smaller to match the planetarium resolution, but context about what is viewed is lost. (c) For learners in Group III, the wide FOV can show widely separated spatial phenomena with no loss of detail. 
By controlling for the virtual environment software and instruction to be as similar as possible in both the classroom and planetarium, we see differences in student gains between the non-immersive and immersive cases. Although this study does not try to unpack the details of how learning occurs for an individual student inside an immersive display, the results here suggest that the egocentric experience of being immersed within the virtual environment is important. In all three test groups, the instructor's verbal lesson complements the displayed visuals, i.e., the static diagrams in Group I, and virtual environments in Groups II and III. But only in Group III does the lecture and the egocentric experience of the immersive virtual simulation reinforce each other. The students do not have to expend cognitive resources to mentally model the Sun and its behavior; they can experience it directly via the immersive virtual environment. Although the instructor in Groups I and II give the same lecture, it is not reinforced by the same visual experience that students in Group III receive.

Group III students also showed better retention of their learning gains after the Post-instruction tests when compared to Group II students. This effect is also seen in Zimmerman et al. (2014) where students who saw an immersive movie had better retention than students who viewed the same movie on their computer screens. It is not clear whether this effect is due to immersion or some other variable, such as the novelty of the immersive display, and should be investigated in future studies.

\section{ACKNOWLEDGMENTS}

This work was supported by the National Science Foundation under grants NSF ROLE 0529522 and DRL 0848945. We thank Jim Dove and Grant Denn for additional misconceptions analysis; Chase Latta for software coding; Doug Howie, Greg Mancari, Dan Neafus for logistical support; Julia Plummer for helpful feedback; and Katherine Honda for assistance with library research.

\section{AUTHOR INFORMATION}

Ka Chun Yu is the Curator of Space Science at DMNS, which he joined in 2001 as part of a team tasked to create planetarium software to visualize the known universe. He continues to create new educational content and visualizations for displays like the digital dome and Science On a Sphere, and research the most effective ways of using this type of technology for education. He is one of the founders of the Worldviews Network, a group using immersive visuals to connect public audiences with global change issues. He currently serves on the International Planetarium Society's Science and Data Visualization Task Force, and IPS' Education Committee. E-mail: kcyu@dmns.org (Contact author)

Kamran Sahami is Professor of Physics at MSUD. His research interests include non-linear systems, electro-optics and physics and astronomy education. He received his Ph.D in Astrophysical, Planetary and Atmospheric Sciences from the University of Colorado in 2001, and also holds two Master of Science degrees in Astrophysics and Applied Physics. At MSUD, he has received the Faculty Senate Excellence in Teaching Award, served as the Interim Director of the Office of Sponsored Research and Programs, and served four terms as President of the Faculty Senate. In Spring 2014, he co-founded the MSUD Chapter of the AAUP and was elected its first President. E-mail: ksahami@msudenver.edu

Victoria Alten Sahami, M.S. is an astronomy instructor at MSUD. She has worked as a solar observer, done research in star formation, and built UV rocket payloads. Currently, she prepares and leads total solar eclipse tours for Sirius Travel in addition to her teaching work. E-mail: vsahami@gmail.com

Larry Sessions, a former planetarium director in Denver and Fort Worth, teaches astronomy at MSUD and the Community College of Aurora, blogs for EarthSky.org, and has a strong interest in promoting public awareness of astronomical events. He was formerly the managing editor at the Denver Museum of Natural History, as well as for the award-winning "Weather Guide" calendar from Accord Publishing. His articles have appeared in numerous publications including Sky \& Telescope, Astronomy and Rolling Stone. E-mail: ast1040@comcast.net 


\section{REFERENCES}

American Association for the Advancement of Science. (2007). Atlas of Science Literacy, Volume 2. Washington, DC: AAAS.

Atwood, R.K. \& Atwood, V.A. (1996). Preservice Elementary Teachers' Conceptions of the Causes of Seasons. Journal of Research in Science Teaching, 53, 553-563.

Bakas, C. \& Mikropoulos, T.A. (2003). Design of virtual environments for the comprehension of planetary phenomena based on students' ideas. International Journal of Science Education, 25, 949-967.

Barnea, N., \& Dori, Y.J. (1999). High-school chemistry students' performance and gender differences in a computerized molecular modeling learning environment. Journal of Science Education and Technology, 8, 257-271.

Baxter, J. (1989). Children's understanding of familiar astronomical events. International Journal of Science Education, 11(5), 502-513.

Baxter, J.H., \& Preece, P.F. (2000). A comparison of dome and computer planetaria in the teaching of astronomy. Research in Science \& Technological Education, 18(1), 63-69.

Bennett, J., Donahue, M., Schneider, N., \& Voit, M. (2007). The Cosmic Perspective, 4/e. San Francisco, CA: Addison-Wesley.

Bisard, W.J., Aron, R.H., Francek, M.A., \& Nelson, B.D. (1994). Assessing selected physical science and earth science misconceptions of middle school through university preservice teachers: Breaking the science 'misconception cycle'. Journal of College Science Teaching, 24(1), 38-42.

Brewer, W.F. (2008). Naïve Theories of Observational Astronomy: Review, Analysis, and Theoretical Implications. In S. Vosniadou (Ed.), International Handbook of Research on Conceptual Change. New York: Routledge.

Chastenay, P. (2015). From Geocentrism to Allocentrism: Teaching the Phases of the Moon in a Digital Full-Dome Planetarium. Research in Science Education, 1-35.

Chen, C.H., Yang, J.C., Shen, S., \& Jeng, M.C. (2007). A desktop virtual reality earth motion system in astronomy education. Educational Technology \& Society, 10(3), 289-304.

Chinn, C. A., \& Brewer, W. F. (1998). An empirical test of a taxonomy of responses to anomalous data in science. Journal of Research in Science Teaching, 35(6), 623-654.

Cohen, J. (1977). Statistical Power analysis for the Behavioral Sciences, Revised Edition, Hillsdale, NJ: Erlbaum.

Cohen, J. (1994). The Earth Is Round (p<.05). American Psychologist. 49(12), 997-1003.

Cumming, G. (2007). Inference by eye: Pictures of confidence intervals and thinking about levels of confidence. Teaching Statistics, 29, 89-93.

Cumming, G., \& Finch, S. (2001). A primer on the understanding, use, and calculation of confidence intervals that are based on central and noncentral distributions. Educational and Psychological Measurement, 61(4), 532574.

Cumming, G., \& Finch, S. (2005). Inference by eye: Confidence intervals, and how to read pictures of data. American Psychologist, 60, 170-180.

Cumming, G., \& Maillardet, R. (2006). Confidence intervals and replication: Where will the next mean fall? Psychological methods, 11(3), 217.

Dede, C. (2000). Emerging Influences of Information Technology on School Curriculum. Journal of Curriculum Studies, 32(2), 281-303.

Emmart, C. (2005). The Powers of Ten with a Steering Wheel on the Global Visualization Superhighway. The Planetarian, 34, 19-26.

Fraser, J., Heimlich, J. E., Jacobsen, J., Yocco, V., Sickler, J., Kisiel, J., \& Stahl, J. (2012). Giant screen film and science learning in museums. Museum Management and Curatorship, 27(2), 179-195.

Gazit, E., Yair, Y., \& Chen, D. (2005). Emerging conceptual understanding of complex astronomical phenomena by using a virtual solar system. Journal of Science Education and Technology, 14(5-6), 459-470.

Hansen, J.A., Barnett, M., MaKinster, J.G., \& Keating, T. (2004). The impact of three-dimensional computational modeling on student understanding of astronomical concepts: a quantitative analysis. International Journal of Science Education, 26(11), 1365-1378.

Heimlich, J.E., Sickler, J., Yocco, V., \& Storksdieck, M. (2010). Influence of Immersion on Visitor Learning: Maya Skies Research Report, Edgewater, MD: Institute for Learning Innovation. 
Heyer, I., Slater, S.J., \& Slater, T.F. (2013). Establishing the Empirical Relationship between Non-science Majoring Undergraduate Learners' Spatial Thinking Skills and Their Conceptual Astronomy Knowledge. Revista Latino-Americana de Educação em Astronomia - RELEA, 16, 45-61.

Hobson, S.M., Trundle, K.C., \& Saçkes, M. (2010). Using a planetarium software program to promote conceptual change with young children. Journal of Science Education and Technology, 19(2), 165-176.

Hufnagel, B. (2002). "Development of the Astronomy Diagnostic Test," Astronomy Education Review, 1(1), 47-51.

Jacobson, J. (2011). Digital Dome vs. Desktop Display in an Educational Game: Gates of Horus.International Journal of Gaming and Computer-Mediated Simulations, 3(1), 13-32.

Johnson, A., Moher, T., Ohlsson, S., \& Gillingham, M. (1999). The round earth project-Collaborative VR for conceptual learning. Computer Graphics and Applications, IEEE, 19(6), 60-69.

Keating, T., Barnett, M., Barab, S.A., \& Hay, K.E. (2002). The virtual solar system project: developing conceptual understanding of astronomical concepts through building three-dimensional computational models. Journal of Science Education and Technology, 11(3), 261-275.

Kikas, E. (1998). The impact of teaching on students' definitions and explanations of astronomical phenomena. Learning and Instruction, 8(5), 439-454.

Kline, R.B. (2004). Beyond Significance Testing. Reforming Data Analysis Methods in Behavioral Research. Washington, DC: APA Books, pp. 61-91.

Kozhevnikov, M., \& Dhond, R.P. (2012). Understanding immersivity: image generation and transformation processes in 3D immersive environments. Frontiers in Psychology, 3, 1-10.

Kozhevnikov, M., \& Garcia, A. (2011). Visual-spatial learning and training in collaborative design in virtual environments. In Collaborative Design in Virtual Environments, Springer Netherlands, pp. 17-26.

Lantz, E. (2011). Planetarium of the Future. Curator, 54(3), 293-312.

Limón, M., \& Carretero, M. (1997). Conceptual change and anomalous data: A case study in the domain of natural sciences. European Journal of Psychology of Education, 12(2), 213-230.

Lochness Productions. (2015). Fulldome Theater Compendium. http://www.lochnessproductions.com/lfco/lfco.html, accessed 12 March 2015.

Lucas, K.B., \& Cohen, M.R. (1999). The changing seasons: Teaching for understanding. Australian Science Teachers Journal, 45(4).

Mason, L. (2001). Introducing talk and writing for conceptual change: A classroom study. Learning and Instruction, $11(4), 305-329$.

Ojala, J. (1992). The third planet. International Journal of Science education, 14(2), 191-200.

Ojala, J. (1997). Lost in space? The concepts of planetary phenomena held by trainee primary school teachers. International Research in Geographical \& Environmental Education, 6(3), 183-203.

Parker, J., \& Heywood, D. (1998). The earth and beyond: developing primary teachers' understanding of basic astronomical events. International Journal of Science Education, 20(5), 503-520.

Peña, B.M., \& Quilez, M.J.G. (2001). The importance of images in astronomy education. International Journal of Science Education, 23(11), 1125-1135.

Plummer, J.D. (2009). Early Elementary Students' Development of Astronomy Concepts in the Planetarium. Journal of Research in Science Teaching, 46(2), 192-209.

Plummer, J. D., \& Krajcik, J. (2010). Building a learning progression for celestial motion: Elementary levels from an earth-based perspective. Journal of Research in Science Teaching, 47(7), 768-787.

Plummer, J.D., \& Maynard, L. (2013). Building a learning progression for celestial motion: An exploration of students' reasoning about the seasons, submitted.

Plummer, J.D., Kocareli, A., \& Slagle, C. (2013). Learning to Explain Astronomy Across Moving Frames of Reference: Exploring the role of classroom and planetarium-based instructional contexts. International Journal of Science Education, 36(7), 1083-1106.

Sadler, P.M. (1998). Psychometric models of student conceptions in science: Reconciling qualitative studies and distractor-driven assessment instruments. Journal of Research in Science Teaching, 35(3), 265-296.

Sadler, P.M., Sonnert, G., Coyle, H.P., Cook-Smith, N., \& Miller, J.L. (2013). The influence of teachers' knowledge on student learning in middle school physical science classrooms. American Educational Research Journal, 50(5), 1020-1049.

Salzman, M. C., Dede, C., \& Loftin, R. B. (1999, May). VR's frames of reference: A visualization technique for mastering abstract multidimensional information. In Proceedings of the SIGCHI conference on Human Factors in Computing Systems. Pittsburgh, PA, pp. 489-495. 
Scott, P.H., Asoko, H.M., \& Driver, R.H. (1992). Teaching for conceptual change: A review of strategies. In R. Duit, F. Goldberg, and H. Niedderer (Eds.), Research in Physics Learning: Theoretical Issues and Empirical Studies, Kiel, Germany: IPN-Leibniz Institute for Science Education, 310-329.

Sneider, C., Bar, V., \& Kavanagh, C. (2011). Learning about seasons: a guide for teachers and curriculum developers. Astronomy Education Review, 10(1), 010103.

Subramaniam, K., \& Padalkar, S. (2009). Visualisation and reasoning in explaining the phases of the moon. International Journal of Science Education, 31(3), 395-417.

Sumners, C., Reiff, P., Weber, W. (2008). Learning In An Immersive Digital Theater. Advances in Space Research, 42, 1848-1854.

Sweller, J., Van Merrienboer, J.J., \& Paas, F.G. (1998). Cognitive architecture and instructional design. Educational Psychology Review, 10(3), 251-296.

Trumper, R. (2000). University students' conceptions of basic astronomy concepts. Physics Education, 35(1), 9-15.

Trundle, K.C., \& Bell, R.L. (2003). Using Planetarium Software to Teach Standards-Based Lunar Concepts. School Science and Mathematics, 103(8), 397-401.

Vosniadou, S., \& Brewer, W. F. (1994). Mental models of the day/night cycle. Cognitive Science, 18(1), 123-183.

Ware, C., \& Osborne, S. (1990). Exploration and virtual camera control in virtual three dimensional environments. In SI3D '90: Proceedings of the 1990 Symposium on Interactive 3D Graphics (pp. 175-183). ACM.

Winn, W., Windschitl, M., Fruland, R., \& Lee, Y. (2002). When does immersion in a virtual environment help students construct understanding. In Proceedings of the International Conference of the Learning Sciences, ICLS, pp. 497-503.

Wyatt, R. (2005). Planetarium paradigm shift. The Planetarian, 34(3), 15-19.

Willard, T. \& Roseman, J.E. (2007). Progression of understanding the reason for seasons. Paper presented at the Knowledge Sharing Institute of the Center for Curriculum Materials in Science, Washington, DC.

Yu, K.C. (2005). Digital Full-Domes: The Future of Virtual Astronomy Education. The Planetarian, 34(3), 6-11.

Yu, K.C., Sahami, K., \& Denn, G. (2010). Student ideas about Kepler's laws and planetary orbital motions. Astronomy Education Review, 9(1), 010108.

Zeilik, M., Schau, C., \& Mattern, N. (1999). Conceptual astronomy. II. Replicating conceptual gains, probing attitude changes across three semesters. American Journal of Physics, 67(10), 923-927.

Zimmerman, L., Spillane, S., Reiff, P., Sumners, C., (2014). Comparison of Student Learning About Space in Immersive and Computer Environments. Journal and Review of Astronomy Education and Outreach, 1(1) A5-A20. 
Seasons Lecture Summary

\section{APPENDIX A}

The seasons lecture follows the recommendations of Willard \& Roseman (2007), and the benchmarks identified by AAAS (2007). The first line of understanding involves the role of sunlight in warming the surface of Earth. To help them build an accurate mental model, students are exposed to a variety of examples of the phenomena of seasons. The lesson begins by showing the diurnal motion of the Sun at different times of the year, and from different latitudes on Earth. This allowed students to become familiar with patterns of the seasons from Earth, before being exposed to space-based perspectives. Students in Groups II and III are introduced to a virtual sky, with cardinal directions (north, south, east, west, etc.) labeled on the horizon. Other graphical markers are used to orient the audience, including the local meridian dividing the sky into eastern and western halves; an altitudeazimuth grid with $10^{\circ}$ increments used to locate objects relative to the camera's location on Earth; and the ecliptic line representing the Sun's annual apparent path. The tilted dome gives the audience unrestricted forward and zenith views of the virtual environment, with the bottom edge of the projection on the left and right sides parallel with the slope of the terraced floor (Figure 2). Even with these restrictions, the visuals convey the experience of viewing the movement of the Sun over the course of a day in real life. The lesson continues by showing how the path of the Sun changes seasonally, to counter the belief that the Sun passes through the zenith when observed from mid-latitudes (Plummer, 2009). Finally to follow Willard \& Roseman's second line of understanding of the role of Earth's motion and orientation with respect to the Sun, the virtual camera is switched between the Earth-based viewpoint of the Sun in the sky with the space-based perspective showing the amount and angle of illumination of Earth by the Sun at different times of the year. Discrepant information is introduced to counter the common belief that the seasons have to do with Earth's distance from the Sun. 


\section{APPENDIX B}

\section{Pre-Instruction}

1a. On which of the following dates is the Sun highest in the sky at midday as seen from Denver?
A. March 20
B. June 21
C. September 22
D. December 21
E. November 20

3b. Flying direct between Denver and New Delhi, India, your private jet loses power and you must make an emergency landing at the North Pole. You notice that the Sun is low to the South. What time of day is it? (Assume that your clocks are not working and your radio and phones don't work,)
A. 3 a.m.
B. 9 a.m.
C. noon
D. 9 p.m.
E. Impossible to tell

4b. Regarding the change in the Seasons, which of the following is a true statement?
A. It gets cold in Winter because the Earth is closer to the Sun then.
B. It gets cold in Winter because the Earth's axis is tilted toward the Sun then.
C. It gets cold in Winter because the Earth is farther from the Sun then.
D. It gets cold in Winter because the Earth's axis is tilted away from the Sun then
E. It gets cold in Winter both because the Earth is farther from the Sun and the axis is tilted away from the Sun

5b. During daylight hours, at what time of day are the shadows cast by the Sun the longest?
A. sunrise
B. mid-morning
C. midday
D. afternoon
E. both sunrise and sunset

6a. During daylight hours, at what time of the year are the shadows cast by the midday Sun the shortest?
A. January
B. March
C. June
D. September
E. November

\section{Contemporaneous}

1a. Twenty-four hours is the average time it takes...
A. the Earth to revolve (orbit) once around the Sun
B. the Sun to revolve once around the Earth
C. the Earth to rotate once on its axis, with respect to the Sun
D. for the stars to revolve once around the Earth
E. (all of these are correct) 
$2 \mathrm{~b}$. On the first day of Summer, the Sun rises to about 73.5 degrees high in Denver. In Calgary on the same day, the Sun rises to about 62.25 degrees high. What could you reasonably conclude from this?
A. That Denver is more than 11 degrees of latitude South of Calgary
B. That Calgary is more than 11 degrees of latitude North of Denver
C. That Denver is more than 11 degrees of latitude North of Calgary
D. That Calgary is nearly 5 degrees of latitude South of Denver
E. (both $\mathrm{A}$ and $\mathrm{B}$ are correct)

4b. On the first day of Winter, Denver received 9 hours and 17 minutes of sunshine. Albuquerque got 9 hours and 43 minutes on the same day. This difference in length of daylight is due to the fact that Albuquerque is
A. East of Denver
B. West of Denver
C. North of Denver
D. South of Denver
E. higher in elevation than Denver

6a. On the December Solstice, Denver received 9 hours and 17 minutes of daylight. On the same day, how much daylight was there at the North Pole?
A. none
B. 6 hours
C. 12 hours
D. 18 hours
E. 24 hours

7a. Which combination best describes the motions of the Sun on the first day of Winter?
A. Early sunrise, high Sun during the day, late sunset
B. Late sunrise, high Sun during the day, early sunset
C. Late sunrise, low Sun during the day, early sunset
D. Early sunrise, low Sun during the day, late sunset
E. Early sunrise, low Sun during the day, early sunset

9a. Which of the following is the best set of facts related to a clear Summer day in Denver?
A. Northern Hemisphere tilted sunward, shorter daylight hours, nearer to Sun than in Winter
B. Farther from Sun than in Winter, Northern Hemisphere tilted sunward, longer daylight hours
C. Nearer to Sun than in Winter, longer daylight hours, Northern Hemisphere tilted away from Sun
D. Farther from Sun than in Winter, Northern Hemisphere tilted away from Sun, shorter daylight hours
E. Longer daylight hours, Northern Hemisphere tilted sunward, nearer to Sun than in Winter

10b. Which of the following is the best set of facts related to a clear Winter day at the North Pole?
A. Northern Hemisphere tilted sunward, 24-hour night, nearer to Sun than in Summer
B. Nearer to Sun than in Summer, 24-hour night, Northern Hemisphere tilted away from Sun
C. Farther from Sun than in Summer, Northern Hemisphere tilted away from Sun, 24-hour night
D. 24-hour day, Northern Hemisphere tilted sunward, nearer to Sun than in Summer
E. Farther from Sun than in Summer, Northern Hemisphere tilted sunward, 24-hour day

11b. Consider that you observe some constellation, say Scorpius, high in the Southern sky tonight at 10 p.m. Approximately where would you have looked to see this same constellation an hour earlier at 9 p.m.?
A. It would be roughly 30 degrees to the West of where it would be at 10 p.m.
B. It would be approximately 90 degrees to the East, near the horizon
C. It would not be visible at all, not having risen yet
D. It would be about 15 degrees to the East of where it was at 10 p.m.
E. It would be in the same position at it would be at 10 p.m. 
12a. Consider that you observe some constellation, say Orion, high in the Southern sky tonight at 10 p.m. Approximately where could you see this same constellation a month from now at 10 p.m.?
A. It would be roughly 30 degrees to the West of where it was tonight at 10 p.m.
B. It would be approximately 90 degrees to the East, near the horizon
C. It would not be visible at all, already having sat
D. It would be about 15 degrees to the East of where it was tonight at 10 p.m.
E. It would be in the same position at it was tonight at 10 p.m.

\section{Post-Instruction}

1b. The rotation of the Earth on its axis causes what?
A. The Seasons
B. The temperature differences between July and January
C. The tilt of the Earth on its axis
D. The daily procession of day and night
E. The gravity that holds us to the planet

$2 \mathrm{~b}$. On average, there are as many hours of darkness as there are of daylight. Why?

A. Because half the time Earth is tilted toward the Sun, half of the time away from the Sun

B. As the Earth rotates, any location faces the Sun half the time and is turned away the other half

C. As the Earth orbits the Sun, half the time we are close to the Sun, and half the time far away

D. Because this balances the hot and cold temperatures, keeping us at a livable level

E. Because Winter requires short days and Summer long days, so day and night average to 12 hours each

3a. A year is about 365 days long, why?
A. That number was chosen because it is almost one degree for every day orbiting the Sun
B. It has to work that way because calendars would not work otherwise
C. 365 days is how long it takes the Sun to rotate on its axis
D. It takes that many days for the Moon to progress through 13 lunar months
E. It's just because as the Earth orbits the Sun once, our planet turns about 365 times on its axis

4a. Which combination of astronomical factors most directly affects temperature on Earth?
A. Length of daylight, speed of Earth in its orbit, global warming induced by pollution
B. Height of Sun in sky, length of daylight, mass of atmosphere through which the Sun shines
C. Earth's average rotational speed, distance to the Sun, length of the year
D. Amount of moonlight at night, cloud cover, Earth's rotational speed
E. Nearness of the Full Moon, length of daylight, length of the year

5a. When the Sun is high in the sky, which of the following sets of circumstances best describe the effects?
A. Long shadows, less direct sunlight, lower temperatures
B. Long shadows, more direct sunlight, lower temperatures
C. Short shadows, more direct sunlight, higher temperatures
D. Short shadows, less direct sunlight, higher temperatures
E. Long shadows, more direct sunlight, higher temperatures 